\title{
Prevalence and Characteristics of Anergia (Lack of Energy) in Patients with Acute Coronary Syndrome
}

\author{
Jonathan A. Shaffer, PhD ${ }^{a, b}$, Karina W. Davidson, PhD $^{a, b}$, Joseph E. Schwartz, PhD ${ }^{a, b, c}$, \\ Daichi Shimbo, MD ${ }^{a, b}$, Jonathan D. Newman, MD, MPH ${ }^{a, b}$, Barry J. Gurland, FRCPhysicians \\ (London) $^{d}$, and Mathew S. Maurer, MD ${ }^{b, d}$ \\ ${ }^{a}$ Center for Cardiovascular Behavioral Health, Columbia University Medical Center, New York, \\ NY

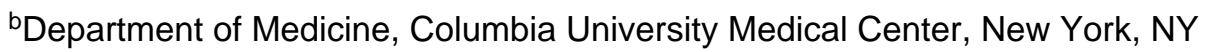 \\ 'Department of Psychiatry, Stony Brook University, New York, NY \\ dStroud Center for the Studies of Quality of Life, Columbia University, New York, NY
}

\begin{abstract}
Anergia, a commonly occurring syndrome in older adults and patients with cardiovascular diseases, is associated with functional and clinical limitations. To date, the prevalence and clinical-demographic characteristics of anergia in patients with acute coronary syndrome (ACS) have not been elucidated. We examined the prevalence and clinical-demographic characteristics of anergia in a multiethnic sample of patients with ACS. Hospitalized patients with ACS $(n=472)$, enrolled in the Prescription Usage Lifestyle and Stress (PULSE) prospective cohort study, completed assessments of demographic, behavioral, and clinical characteristics within 7 days of hospitalization for an ACS event. Current depressive disorder was ascertained using a structured psychiatric interview 3 to 7 days post-discharge. Anergia was assessed at baseline and defined using patients' binary responses (yes/no) to seven items related to energy level. At least 1 complaint of anergia was reported by $79.9 \%(n=377)$ of patients, and $32 \%(n=153)$ of patients met criteria for anergia. In a multivariable logistic regression model, anergia was independently associated with being female, white (compared to black), having bodily pain, participating in exercise, having current depressive disorder, and having higher values on the Charlson comorbidity index. In conclusion, anergia is a highly prevalent syndrome among patients with ACS. It is distinct from depression and is associated with modifiable clinical factors such as participation in exercise and bodily pain that may be appropriate targets for intervention.
\end{abstract}

\section{Keywords}

anergia; acute coronary syndrome; signs and symptoms

\section{(C) 2012 Excerpta Medica, Inc. All rights reserved.}

Corresponding Author: Mathew S. Maurer, M.D. Clinical Cardiovascular Research Laboratory for the Elderly, Allen Pavilion of New York Presbyterian Hospital, 5141 Broadway, New York, NY 10034 Telephone: 212-305-9808; Fax: 212-932-4538; msm10@columbia.edu.

Publisher's Disclaimer: This is a PDF file of an unedited manuscript that has been accepted for publication. As a service to our customers we are providing this early version of the manuscript. The manuscript will undergo copyediting, typesetting, and review of the resulting proof before it is published in its final citable form. Please note that during the production process errors may be discovered which could affect the content, and all legal disclaimers that apply to the journal pertain. 
Among the chronic symptoms reported by patients with coronary artery disease and other chronic health conditions, fatigue is among the most common and is strongly associated with negative outcomes. ${ }^{1-2}$ Anergia (i.e., lack of energy) is a recently delineated criterionbased syndrome that is conceptually analogous to fatigue. ${ }^{3-4}$ Unlike fatigue, however, anergia is conceptualized to be more persistent and not specifically post-exertional. Although fatigue as a symptom of ACS has been studied by some investigators ${ }^{5-6}$, to date, the prevalence and clinical, demographic, and behavioral characteristics of anergia in patients with acute coronary syndrome (ACS) have not been investigated. We therefore sought to evaluate the prevalence of anergia in a multiethnic sample of participants with ACS; to delineate the clinical-demographic characteristics of participants with anergia compared to those without anergia; and to more fully examine the relationship between anergia and depression among patients with ACS. We hypothesized that anergia: (1) would be a prevalent condition among participants with ACS; (2) would be strongly associated with clinically modifiable factors; and (3) would be sufficiently distinct from depression to warrant ongoing clinical investigation in its own right.

\section{Methods}

Participants were hospitalized patients with ACS enrolled in the Prescription Usage Lifestyle and Stress (PULSE) study, a prospective cohort study of the prognostic risk conferred by depression at the time of an ACS. Patients with unstable angina pectoris (UA) or acute ST and non-ST segment elevation myocardial infarction (MI) were recruited from Columbia University Medical Center within 1 week of hospitalization for their ACS. Patients completed a structured psychiatric interview 3 to 7 days post-discharge to ascertain their depression status, and they returned for a follow-up visit 1 month later. The current analyses include 472 participants who completed the self-report anergia questionnaire during the baseline (in-hospital) interview. Excluded from bivariate analyses were 28 participants with missing data on anergia at baseline. Data collection occurred between February 2009 and June 2010. The Institutional Review Board of Columbia University approved this study, and all participants provided informed consent.

Using a questionnaire that has been included in previous studies, ${ }^{3,4}$ anergia was defined by participants' binary responses (yes/no) to 7 items related to energy level. It was operationalized as the presence of the cardinal criterion "Sits around a lot for lack of energy" and any 2 of the 6 following additional criteria: "recently not enough energy," "felt slowed physically in the past month," "doing less than usual in the past month," "any slowness is worse in the morning," "wakes up feeling tired," and/or "naps (> 2 hours) during the day." Participants completed this questionnaire, which has acceptable internal reliability, face validity, and predictive validity for morbidity and mortality,4 during their in-hospital interview.

At baseline, participants identified their ethnicity (Hispanic versus non-Hispanic), race (white versus black versus other), years of education, partner status, participation in regular exercise, and cigarette smoking status. Medical charts were used to ascertain prior cardiovascular disease and cardiac procedures (angina pectoris, MI, percutaneous coronary intervention [PCI], coronary artery bypass grafting [CABG], New York Heart Association [NYHA] heart failure class), prior cerebrovascular disease (stroke, transient ischemic attack), and other chronic medical conditions (respiratory diseases, liver diseases, rheumatologic diseases, and stomach ulcers). Age and gender were recorded for each patient. The Global Registry of Acute Coronary Events (GRACE) risk score was used to calculate 6-month post-ACS mortality risk, ${ }^{7}$ and medical comorbidities were assessed using the Charlson comorbidity index. ${ }^{8}$ 
Blood samples were collected from a subset of participants $(n=259)$, and serum concentrations of C-reactive protein (hs-CRP) were determined using a high-sensitivity enzyme-linked immunoabsorbent assay. Left ventricular ejection fraction was assessed by ECG, ventriculography, or nuclear stress testing. ACS type (UA, ST-segment MI, non-STsegment MI) was determined from chart review by study cardiologists. In-hospital hematocrit (\%), thyroid stimulating hormone, and serum creatinine at admission were ascertained from patient charts and used to determine anemia status, ${ }^{9}$ thyroid function, ${ }^{10}$ and estimated glomerular filtration rate (eGFR). ${ }^{11}$ Probable chronic kidney disease was defined as an eGFR $<60 \mathrm{ml} / \mathrm{min} / 1.73 \mathrm{~m}^{2}$, and anemia was defined as a hematocrit level $<36 \%$ for women and $<39 \%$ for men. ${ }^{12}$

Participants were evaluated for symptoms of depression at baseline based on their responses to the 21-item Beck Depression Inventory (BDI) ${ }^{13}$ and the 9-item Patient Health Questionnaire (PHQ-9). ${ }^{14}$ Symptoms of anxiety were determined based on participants' responses to the Hospital Anxiety and Depression Scale-anxiety subscale (HADS-A). ${ }^{15}$

Three to 7 days following their discharge from the hospital, participants were telephoned and evaluated by a trained mental health professional for a depressive disorder using the Diagnostic Interview and Structured Hamilton (DISH), a gold-standard, structured interview that was developed to screen cardiac patients for depressive disorders. ${ }^{16}$ The DISH is used to diagnose major and minor depression according to criteria in the Fourth Edition of the Diagnostic and Statistical Manual of Mental Disorders (DSM-IV). ${ }^{17}$ Consistent with other studies of depression among patients with ACS, ${ }^{18}$ diagnostic criteria were modified such that participants received a diagnosis if they met the symptom criteria for a major or minor depressive episode for at least 7 (instead of the usual 14) days or if they reported taking antidepressant medication. Participants who did not complete the DISH but who met criteria for a major depressive or other depressive syndrome on the PHQ-9 according to a validated diagnostic algorithm, ${ }^{19}$ were considered to have a current depressive disorder. Current and past depressive disorders were identified separately using time periods included in the DISH.

One-month following their discharge from the hospital, participants returned for a follow-up visit during which they completed the Pittsburgh Sleep Quality Inventory (PSQI), a measure that has been used to identify individuals with poor sleep quality. ${ }^{20}$ Poor sleep quality was defined categorically as a global PSQI score $>5$, a cutoff with acceptable diagnostic sensitivity and specificity. ${ }^{21}$ Participants also completed a pain item from the Short Form Health Survey (SF-12), which asks "how much did pain interfere with your normal work (... both work outside the home and housework). ${ }^{21}$ Responses are provided on a 5-point scale (1, "not at all" to 5, "extremely") and converted to T-scores using normative data. Higher Tscores indicate less pain.

The prevalence of anergia and its components was determined. To investigate the associations of anergia with clinical-demographic measures, we performed bivariate analyses comparing those with and without anergia. Chi-square $\left(\chi^{2}\right)$ analyses with Fisher's exact test were used for dichotomous variables and independent samples $t$-tests were used for continuous variables. A multivariable logistic regression model was estimated to ascertain which clinical-demographic variables were independently associated with anergia. This model included all variables that were significant at $p<0.10$ in bivariate analysis as well as any clinically relevant variables identified a priori (e.g., age, gender, race, and partner status). Multiple imputation using a fully conditional specification method was performed to account for missing data in the multivariable logistic regression model. Data are presented as odds ratios and 95\% confidence intervals. A two-tailed $p$ value $<0.05$ was considered statistically significant. 
The PULSE study includes several measures of depression (BDI, PHQ-9, current depressive disorder, and past depressive disorder), and a primary aim of the current analyses was to further elucidate the association of anergia with depression. Given that the strong intercorrelations among these depression measures would affect parameter estimates in any multivariable regression analyses that included them all simultaneously, we decided a priori to include current depressive disorder as the measure of depression in our multivariable model, as it is based upon the gold-standard DISH assessment for depression diagnosis. Sensitivity analyses were subsequently conducted in which we substituted the BDI and PHQ-9 for current depressive disorder in the multivariable model. Because history of several of the cardiovascular and non-cardiac diseases is captured in the Charlson comorbidity score, we likewise made the a priori decision to include only the Charlson comorbidity score in our multivariable model.

Given the strong association between depression and anergia reported in a previous study 3 and the strong resemblance of depressive symptoms and symptoms of anergia, we further sought to identify clinical-demographic factors that accompany anergia among those without a concurrent depressive disorder. To do so, we performed bivariate analyses comparing those with and without anergia, among participants without a depressive disorder. Because the distribution of hs-CRP concentration was not normal, this variable was log-transformed for all analyses. All analyses were performed in SPSS 18.0.22

\section{Results}

The prevalence of the criteria used to identify participants with anergia in the PULSE cohort are shown in Table 1. At least one complaint of anergia was reported by $79.9 \%(n=377)$ of participants at baseline, and 32\% $(n=153)$ of participants met criteria for anergia at baseline.

The clinical-demographic characteristics of participants, stratified by anergia status at baseline, are shown in Table 2. Participants' ages ranged from 26 to 96 years. The cohort included a multiethnic population of predominately non-Hispanic participants, though participants of Hispanic ethnicity constituted approximately $32 \%$ of the sample. The majority of participants was white, male, and partnered or married. The most common type of ACS with which participants presented to the hospital was unstable angina pectoris.

The proportion of women was significantly greater among those with anergia than among those without anergia. Those with anergia were more likely to be without a partner or spouse, and were more likely to report being active smokers compared to those without anergia. In contrast, those with anergia were less likely to participate in regular exercise. Anergia at the time of the ACS was not significantly associated with age, race, or ethnicity.

Anergia was associated with worse physical functioning, prior cardiovascular diseases and cardiac procedures, and several chronic medical diseases (Table 2). Participants with anergia were significantly more likely to have poor sleep quality, chronic kidney disease (as assessed by an eGFR < 60), liver diseases, rheumatologic diseases, and stomach ulcers compared to those without anergia. History of cardiovascular diseases and procedures, including angina pectoris, MI, PCI, CABG, and heart failure were also more common among those with versus without anergia. Moreover, those with anergia had significantly more severe heart failure symptoms, as measured by the NYHA class, had higher Charlson comorbidity scores, and reported greater bodily pain compared to those without anergia. Anergia was not significantly associated with type of index ACS event, history of cerebrovascular diseases, left ventricular ejection fraction, anemia, thyroid function, GRACE risk score, or hs-CRP. 
Anergia was associated with all psychosocial factors, including depressive symptom severity (as measured by both the BDI and PHQ-9), current and past depressive disorders, and symptoms of anxiety. Although $31 \%$ of participants with anergia had a current depressive disorder, approximately half of those with anergia $(n=78)$ had never had a depressive disorder, and only $42 \%$ of those who had ever had a depressive disorder had anergia. In addition, the bivariate association between the BDI and anergia status was significant but modest $\left(r^{2}=0.19\right)$. This association was further attenuated $\left(r^{2}=0.17\right)$ after eliminating two BDI items regarding sleep and tiredness from the overall score.

Multivariable logistic regression was used to identify factors that were independently associated with anergia, immediately post-ACS (Table 3). These included being female, white (compared to black), having bodily pain, participating in regular exercise, and having current depressive disorder. Female participants had approximately twice the odds of having anergia as males. Black participants were less likely to be anergic than whites, independent of other risk factors. Those with a current depressive disorder had 1.9 times the odds of having anergia as those without a current depressive disorder. Those who did not participate in regular exercise were also more likely to have anergia than those who did. Bodily pain, as measured by the SF-12 bodily pain T-score, was also independently associated with risk for anergia such that those with higher scores on the bodily pain index, indicating less bodily pain, were significantly less likely to be anergic. Each standard deviation increase in the SF-12 bodily pain T-score was associated with approximately $40 \%$ lower odds of having anergia.

Sensitivity analyses, in which both the BDI and PHQ-9 were substituted for current depressive disorder in the multivariable model, revealed that both measures of depressive symptom severity were significantly and independently associated with anergia. Each standard deviation increase on the PHQ-9 was associated with a 3.21 greater odds of having anergia at baseline $(\mathrm{OR}=3.21,95 \% \mathrm{CI}=2.27-4.55, p<0.001)$, while each standard deviation increase on the BDI was associated with a 2.33 greater odds of having anergia at baseline $(\mathrm{OR}=2.33,95 \% \mathrm{CI}=1.68-3.24, p<0.023)$. When both the BDI and PHQ-9 were included in the same multivariable model, each remained significant, suggesting that may have distinct clinical implications with respect to the prediction of anergia.

To identify which clinical-demographic characteristics differentiate those with versus without anergia among those without a concurrent depressive disorder we conducted additional analyses. Of the 394 participants without a current depressive disorder, approximately $27 \%$ had anergia $(n=106)$. Consistent with the analyses of the full cohort reported above, those with anergia in this subsample were significantly more likely to be female and single. They were also significantly more likely to report poor sleep quality, to have liver and rheumatologic diseases, to have heart failure, prior angina pectoris, and prior $\mathrm{CABG}$, and to have chronic kidney disease. They were significantly less likely to participate in regular exercise, reported significantly greater bodily pain, and had significantly higher Charlson comorbidity indices and Grace risk scores. Although participants with anergia in this subsample did not have a current depressive disorder, they nonetheless reported significantly greater depressive symptoms on both the BDI and PHQ; these patients also reported significantly greater anxiety symptoms on the HADS-A.

\section{Discussion}

The principal findings of this investigation are that anergia: (1) is a common syndrome among post-ACS patients; (2) is associated with several behaviors and clinical characteristics, and (3) is distinct from depression. Despite the strong association and conceptual similarities between anergia and depression, only half of participants with 
anergia had ever had a depressive disorder and only $42 \%$ of those who had ever had a depressive disorder had anergia.

Our findings differ from those of other studies in that anergia was not associated with age in either unadjusted or adjusted analyses. Indeed, supplemental analyses (not shown) in which age was categorized in multiple ways failed to reveal any associations of age with either individual components of anergia, number of anergia complaints, or anergia status. In contrast, a multiethnic cohort study of community-dwelling older adults ${ }^{3}$ found that anergia was strongly associated with older age. However, that study included a sample of Medicare beneficiaries who were $\geq 65$ years of age and who were not evaluated in the context of an acute hospitalization. Accordingly, we hypothesize that the acute cardiovascular event experienced by participants in the current study may explain the lack of an age effect on the prevalence of anergia in this population. In support of this hypothesis, the prevalence of anergia in this study was higher $(32 \%)$ than the prevalence found in the population-based study (18\%), despite the fact that those in the previous study were, on average, 10 years older than those in the current cohort.

Also unique to our study is the finding--based on self-report of race--that black participants were less likely to have anergia than those who identified as being white. Although the aforementioned population-based study of anergia found that non-Hispanic, white participants had non-significantly elevated odds of having anergia compared to Hispanic participants, that study also found that black participants had significantly elevated odds of having anergia compared to Hispanics. Given the different categorizations of race-ethnicity in our study and the previous population-based study, these differences may not be directly comparable. Although early reports of racial-ethnic differences in Chronic Fatigue Syndrome, which is also characterized by a lack of energy and tiredness, suggested that this disorder is more common among whites, more recent reports have found that being black is associated with both a greater risk of Chronic Fatigue Syndrome and with greater severity of symptoms. ${ }^{23}$ Additional research may be warranted to clarify the associations between raceethnicity and anergia.

While anergia is one of the most common concerns expressed by patients with ACS, it is currently not a traditional target for intervention. Our data suggest that certain conditions and behaviors have independent residual associations with anergia and that most of these are potentially modifiable. Using a traditional clinical approach of generating a differential diagnosis based on the frequency and severity of underlying causes contributing to a manifest concern, we would suggest that bodily pain, depression, and lack of participation in regular exercise all have sufficient frequency in patients with ACS and anergia and independent associations with anergia (Table 2) so as to warrant further evaluation at present. Interventions that aim to address chronic pain (e.g. pharmacologic therapy, ${ }^{24}$ relaxation training, ${ }^{25}$ and mindfulness meditation ${ }^{26,27}$ ), decrease depression (e.g., cognitivebehavioral therapy ${ }^{28}$ ), and/or increase participation in exercise (e.g., cardiac rehabilitation29 and motivational/volitional interventions ${ }^{30}$ ) may thus prove efficacious for those ACS patients with anergia.

Notably our data suggest that comorbid conditions that are commonly evaluated in patients with lack of energy, including anemia and thyroid dysfunction, are not strongly associated with anergia in those with ACS. Additionally, the lack of difference in CRP between those with and those without anergia does not suggest a role for chronic low-grade inflammation as a correlate of anergia in patients with ACS. Accordingly, evaluation of these clinical concerns (in addition to rheumatologic diseases and chronic kidney disease) as a cause or contributing factor to anergia should be reserved for circumstances in which other common correlates of anergia have been formally evaluated and ruled out. 
Notwithstanding the PULSE study's rigorous assessment of biopsychosocial risk factors and the ascertainment of a multiethnic cohort of patients with ACS, some limitations remain. Perhaps of greatest importance, our analyses were cross-sectional and preclude conclusions regarding causal paths between clinical-demographic factors and anergia. Given that the PULSE study includes repeat assessments of several clinical-demographic variables, future analyses will consider prospective associations among anergia, behavioral variables, and cardiac outcomes.

\section{Acknowledgments}

Funding \& Support: This work was supported by grants HL-088117, HL-076857, HL-080665, HL-101663, and HL-084034 from the National Heart, Lung, and Blood Institute; by grant T32 HP10260 from the Health Resources and Services Administration; and by grant 12CRP8870004 from the American Heart Association. Dr. Maurer is supported by grant K24-AG036778 from the National Institute of Aging.

\section{References}

1. Evans E, Wickstrom B. Subjective fatigue and self-care in individuals with chronic illness. Medsurg Nurs. 1999; 8:363-369. [PubMed: 11000775]

2. Falk K, Swedberg K, Gaston-Johansson F, Ekman I. Fatigue is a prevalent and severe symptom associated with uncertainty and sense of coherence in patients with chronic heart failure. Eur J Cardiovasc Nurs. 2007; 6:99-104. [PubMed: 16831569]

3. Cheng H, Gurland BJ, Maurer MS. Self-reported lack of energy (anergia) among elders in a multiethnic community. J Gerontol A: Biol Sci Med Sci. 2008; 63:707-714. [PubMed: 18693225]

4. Maurer MS, Cuddihy P, Weisenberg J, Delisle S, Strong BM, Gao Q, Kachnowski S, Howell J. The prevalence and impact of anergia (lack of energy) in subjects with heart failure and its associations with actigraphy. J Card Fail. 2009; 15:145-151. [PubMed: 19254674]

5. Maynard C, Weaver WD. Treatment of women with acute MI: new findings from the MITI registry. J Myocard Ischemia. 1992; 4:27-37.

6. Penque S, Halm M, Smith M, Deutsch J, Van Roekel M, McLaughlin L, Dzubay S, Doll N, Beahrs M. Women and coronary disease: Relationship between descriptors of signs and symptoms and diagnostic treatment course. Am J Crit Care. 1998; 7:175-182. [PubMed: 9579242]

7. Granger CB, Goldberg RJ, Dabbous O, Pieper KS, Eagle KA, Cannon CP, Van De Werf F, Avezum A, Goodman SG, Flather MD, Fox KA. Global Registry of Acute Coronary Events Investigators. Predictors of hospital mortality in the global registry of acute coronary events. Arch Intern Med. 2003; 163:2345-2353. [PubMed: 14581255]

8. Charlson M, Szatrowski TP, Peterson J, Gold J. Validation of a combined comorbidity index. J Clin Epidemiol. 1994; 47:1245-1251. [PubMed: 7722560]

9. McLean E, Cogswell M, Egli I, Wojdyla D, De Benoist B. Worldwide prevalence of anaemia, WHO vitamin and mineral nutrition information system, 1993-2005. Public Health Nutr. 2009; 12:444454. [PubMed: 18498676]

10. Rodondi N, Bauer DC, Cappola AR, Cronuz J, Robbins J, Fried LP, Ladenson PW, Vittinghoff E, Gottdiener JS, Newman AB. Subclinical thyroid dysfunction, cardiac function, and the risk of heart failure: The Cardiovascular Health Study. J Am Coll Cardiol. 2008; 52:1152-1159. [PubMed: 18804743]

11. Poggio ED, Wang X, Greene T, Van Lente F, Hall PM. Performance of the modification of diet in renal disease and Cockcroft-Gault equations in the estimation of GFR in health and in chronic kidney disease. J Am Soc Nephrol. 2005; 16:459-466. [PubMed: 15615823]

12. World Health Organization Scientific Group. Nutritional anaemias: Report of a WHO Scientific Group. World Health Organization; 1968.

13. Beck AT, Ward CH, Mendelson M, Mock J, Erbaugh J. An inventory for measuring depression. Arch Gen Psych. 1961; 4:561-571.

14. Spitzer RL, Kroenke K, Williams JBW. Validation and utility of a self-report version of PRIMEMD. JAMA. 1999; 282:1737-1744. [PubMed: 10568646] 
15. Zigmond AS, Snaith R. The hospital anxiety and depression scale. Acta Psychiatr Scand. 1983; 67:361-370. [PubMed: 6880820]

16. Freedland KE, Skala JA, Carney RM, Raczynski JM, Taylor CB, Mendes de Leon CF, Ironson G, Youngblood ME, Krishnan KR, Veith RC. The Depression Interview and Structured Hamilton (DISH): Rationale, development, characteristics, and clinical validity. Psychosom Med. 2002; 64:897-905. [PubMed: 12461195]

17. American Psychaitric Assocation. Diagnostic and Statistical Manual of Mental Disorders (DSMIV). Washington, DC: 1994.

18. ENRICHD Investigators. Enhancing Recovery in Coronary Heart Disease (ENRICHD) study intervention: rationale and design. Psychosom Med. 2001; 63:747-755. [PubMed: 11573023]

19. Kroenke K, Spitzer RL, Williams JBW. The PHQ-9. J Gen Intern Med. 2001; 16:606-613. [PubMed: 11556941]

20. Buysse DJ, Reynolds CF III, Monk TH, Berman SR, Kupfer DJ. The Pittsburgh Sleep Quality Index: A new instrument for psychiatric practice and research. Psychiat Res. 1989; 28:193-213.

21. Ware JE Jr, Kosinski M, Keller SD. A 12-Item Short-Form Health Survey: Construction of scales and preliminary tests of reliability and validity. Med Care. 1996; 34:220-233. [PubMed: 8628042]

22. SPSS Inc. Statistical Package for Social Sciences, Version 18.0. Chicago, Ill: SPSS Inc.; 2009.

23. Jason LA, Richman JA, Rademaker AW, Jordan KM, Plioplys AV, Taylor RR, McCready W, Huang CF, Plioplys S. A community-based study of chronic fatigue syndrome. Arch Intern Med. 1999; 159:2129-2137. [PubMed: 10527290]

24. Kroenke K, Krebs EE, Bair MJ. Pharmacotherapy of chronic pain: A synthesis of recommendations from systematic reviews. Gen Hosp Psychiatry. 2009; 31:206-219. [PubMed: 19410099]

25. Turner JA, Chapman CR. Psychological interventions for chronic pain: A critical review. I. Relaxation training and biofeedback. Pain. 1982; 2:1-21. [PubMed: 7036049]

26. Kabat-Zinn J, Lipworth L, Burney R. The clinical use of mindfulness meditation for the selfregulation of chronic pain. J Behav Med. 1985; 8:163-190. [PubMed: 3897551]

27. Kabat-Zinn J. An outpatient program in behavioral medicine for chronic pain patients based on the practice of mindfulness meditation: Theoretical considerations and preliminary results. Gen Hosp Psychiatry. 1982; 4:33-47. [PubMed: 7042457]

28. Kroenke K, Swindle R. Cognitive-behavioral therapy for somatization and symptom syndromes: A critical review of controlled clinical trials. Psychother Psychosom. 2000; 69:205-215. [PubMed: 10867588]

29. Carlson JJ, Johnson JA, Franklin BA, VanderLaan RL. Program participation, exercise adherence, cardiovascular outcomes, and program cost of traditional versus modified cardiac rehabilitation. Am J Cardiol. 2000; 86:17-23. [PubMed: 10867086]

30. Milne S, Orbell S, Sheeran P. Combining motivational and volitional interventions to promote exercise participation: Protection motivation theory and implementation intentions. Bri J Health Psych. 2002; 7:163-184. 


\section{Table 1}

Prevalence of components used to define anergia among 472 patients with acute coronary syndrome

\begin{tabular}{ll}
\hline Prevalence of Anergia Components & Number of Participants \\
\hline Recently not enough energy & $242(51.3 \%)$ \\
Felt slowed physically in a month & $270(57.2 \%)$ \\
Doing less than usual in a month & $190(40.3 \%)$ \\
Any slowness worse in the morning & $94(19.9 \%)$ \\
*Sits around a lot for lack of energy (cardinal criterion) & $169(35.8 \%)$ \\
Wakes up feeling tired & $192(40.7 \%)$ \\
Naps during the day & $41(8.7 \%)$ \\
\hline
\end{tabular}


Table 2

Clinical-demographic characteristics of 472 patients with acute coronary syndrome, stratified by anergia status

\begin{tabular}{|c|c|c|c|c|}
\hline \multirow[b]{2}{*}{ Characteristic } & \multicolumn{4}{|c|}{ Anergia } \\
\hline & $\begin{array}{c}\text { Total } \\
(n=472)\end{array}$ & $\begin{array}{c}\text { Yes } \\
(n=153)\end{array}$ & $\begin{array}{c}\text { No } \\
(n=319)\end{array}$ & $\mathbf{P}$ \\
\hline Age (years), $M \pm \mathrm{SD}$ & $63.2 \pm 11.4$ & $63.4 \pm 10.9$ & $63.2 \pm 11.7$ & 0.86 \\
\hline Women & $162(34.3 \%)$ & $78(51.0 \%)$ & $84(26.3 \%)$ & $<0.0001$ \\
\hline Ethnicity (Hispanic) & $152(32.2)$ & $49(32.0)$ & $103(32.3)$ & 0.95 \\
\hline White & $288(61.4 \%)$ & $101(66.0 \%)$ & $187(59.2 \%)$ & 0.34 \\
\hline Black & $94(20.0 \%)$ & $26(17.0 \%)$ & $68(21.5 \%)$ & \\
\hline Other & $87(18.6 \%)$ & $26(17.0 \%)$ & $61(19.3 \%)$ & \\
\hline Education (years), $M \pm \mathrm{SD}$ & $13.3 \pm 4.1$ & $12.9 \pm 3.6$ & $13.5 \pm 4.3$ & 0.12 \\
\hline No partner/spouse & $187(39.9 \%)$ & $76(50.3 \%)$ & $111(34.9 \%)$ & 0.001 \\
\hline Partner/spouse & $282(60.1 \%)$ & $75(49.7 \%)$ & $207(65.1 \%)$ & \\
\hline Unstable angina pectoris & $288(61.0 \%)$ & $95(62.1 \%)$ & $193(60.5 \%)$ & 0.85 \\
\hline ST-segment myocardial infarction & $55(11.7 \%)$ & $16(10.5 \%)$ & $39(12.2 \%)$ & \\
\hline Non-ST-segment myocardial infarction & $129(27.3 \%)$ & $42(27.5 \%)$ & $87(27.3 \%)$ & \\
\hline \multicolumn{5}{|l|}{ Prior CVD and procedures } \\
\hline Angina pectoris & $291(62.2 \%)$ & $107(70.4 \%)$ & $184(58.2 \%)$ & 0.011 \\
\hline Myocardial infarction & $133(28.4 \%)$ & $54(35.5 \%)$ & $79(24.9 \%)$ & 0.017 \\
\hline Percutaneous coronary intervention & $216(46.4 \%)$ & $81(54.0 \%)$ & $135(42.7 \%)$ & 0.023 \\
\hline Coronary artery bypass grafting & $88(18.8 \%)$ & $44(28.9 \%)$ & $44(13.9 \%)$ & $<0.001$ \\
\hline \multicolumn{5}{|l|}{ Prior cerebrovascular disease } \\
\hline Stroke & $20(4.3 \%)$ & $8(5.3 \%)$ & $12(3.8 \%)$ & 0.46 \\
\hline Transient ischemic attack & $20(4.3 \%)$ & $10(6.8 \%)$ & $10(3.2 \%)$ & 0.08 \\
\hline Prior heart failure & $51(10.9 \%)$ & $27(17.8 \%)$ & $24(7.6 \%)$ & 0.001 \\
\hline New York Heart Association class, $M \pm \mathrm{SD}$ & $2.1 \pm 0.9$ & $2.4 \pm 0.9$ & $1.7 \pm 0.7$ & 0.01 \\
\hline Left ventricular ejection fraction (\%), $M \pm \mathrm{SD}$ & $50.4 \pm 11.6$ & $49.9 \pm 12.3$ & $50.6 \pm 11.3$ & 0.56 \\
\hline Respiratory diseases & $58(12.3 \%)$ & $24(15.7 \%)$ & $34(10.7 \%)$ & 0.12 \\
\hline Liver diseases & $7(1.5 \%)$ & $5(3.3 \%)$ & $2(0.6 \%)$ & 0.038 \\
\hline Rheumatologic diseases & $38(8.1 \%)$ & $26(17.0 \%)$ & $12(3.8 \%)$ & $<0.0001$ \\
\hline Stomach ulcers & $25(5.3 \%)$ & $14(9.2 \%)$ & $11(3.4 \%)$ & 0.014 \\
\hline High-sensitivity C-reactive protein (mg/dL), $M \pm \mathrm{SD}$ & $1.8 \pm 1.4$ & $1.8 \pm 1.3$ & $1.8 \pm 1.4$ & 0.99 \\
\hline Anemia $^{a}$ & $157(48.5 \%)$ & $55(53.4 \%)$ & $102(46.2 \%)$ & 0.22 \\
\hline Estimated glomerular filtration rate $<60 \mathrm{ml} / \mathrm{min} / 1.73^{2}$ & $118(26.2 \%)$ & $48(32.7 \%)$ & $70(23.0 \%)$ & 0.029 \\
\hline Subclinical hyperthyroidism & $6(2.1 \%)$ & $4(4.4 \%)$ & $2(1.0 \%)$ & 0.13 \\
\hline Euthyroid & $251(89.0 \%)$ & $82(90.1 \%)$ & $169(88.5 \%)$ & \\
\hline Moderate hypothyroidism & $22(7.8 \%)$ & $5(5.5 \%)$ & $17(8.9 \%)$ & \\
\hline Severe hypothyroidism & $3(1.1 \%)$ & 0 & $3(1.6 \%)$ & \\
\hline Grace risk score, $M \pm \mathrm{SD}$ & $90.2 \pm 29.4$ & $93.6 \pm 30.3$ & $88.6 \pm 28.8$ & 0.085 \\
\hline Charlson comorbidity index, $M \pm \mathrm{SD}$ & $1.7 \pm 1.6$ & $2.2 \pm 1.9$ & $1.4 \pm 1.4$ & $<0.001$ \\
\hline Participation in regular exercise & $208(44.4 \%)$ & $50(33.1 \%)$ & $158(49.8 \%)$ & 0.001 \\
\hline Current smoker & $73(15.5 \%)$ & $31(20.3 \%)$ & $42(13.2 \%)$ & 0.046 \\
\hline
\end{tabular}




\begin{tabular}{lcccc}
\hline & \multicolumn{4}{c}{ Anergia } \\
Characteristic & $\begin{array}{c}\text { Total } \\
(\boldsymbol{n = 4 7 2})\end{array}$ & $\begin{array}{c}\text { Yes } \\
(\boldsymbol{n = 1 5 3})\end{array}$ & $\begin{array}{c}\text { No } \\
(\boldsymbol{n = 3 1 9 )}\end{array}$ & \multicolumn{1}{c}{ P } \\
\hline Poor sleep quality (PSQI 25$)$ & $180(46.9 \%)$ & $80(62.0 \%)$ & $100(39.2 \%)$ & $<0.0001$ \\
Current depressive disorder & $81(17.2 \%)$ & $48(31.4 \%)$ & $33(10.3 \%)$ & $<0.0001$ \\
Current or past depressive disorder & $213(45.1 \%)$ & $91(59.5 \%)$ & $122(38.2 \%)$ & $<0.0001$ \\
Beck Depression Inventory, $M \pm$ SD (Range: 0-63) & $9.2 \pm 7.3$ & $13.9 \pm 8.2$ & $7.0 \pm 5.7$ & $<0.0001$ \\
Patient Health Questionnaire, $M \pm$ SD (Range: 0-27) & $4.4 \pm 4.8$ & $7.9 \pm 5.5$ & $2.8 \pm 3.4$ & $<0.0001$ \\
HADS-A (Range: 0-21) & $3.1 \pm 3.3$ & $4.7 \pm 4.0$ & $2.3 \pm 2.6$ & $<0.0001$ \\
Probable anxiety disorder (HADS-A $\geq 8)$ & $48(11.2 \%)$ & $30(21.4 \%)$ & $18(6.3 \%)$ & $<0.0001$ \\
Bodily pain, $M \pm$ SD $b$ & $45.3 \pm 12.5$ & $39.2 \pm 13.7$ & $48.2 \pm 10.8$ & $<0.001$ \\
\hline
\end{tabular}

${ }^{a}$ Anemia was defined using the sex-specific hemoglobin thresholds used by the World Health Organization to classify persons living at sea level as anemic. $^{12}$

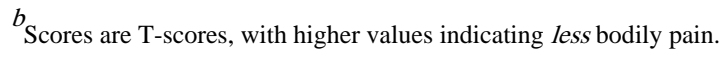

Abbreviations: Pittsburgh Sleep Quality Inventory; HADS-A, Hospital Anxiety and Depression scale—Anxiety subscale. 
Table 3

Multivariable associations between clinical-demographic characteristics and baseline anergia among 500 patients with acute coronary syndrome

\begin{tabular}{lcc}
\hline Parameter & Adjusted OR (95\% CI) & P \\
\hline Age (years) ${ }^{a}$ & $0.80(0.51-1.24)$ & 0.25 \\
Gender (Reference = Male) & $1.86(1.13-3.07)$ & 0.015 \\
Race (Reference $=$ White) & & \\
$\quad$ Black & $0.39(0.21-0.73)$ & 0.003 \\
$\quad$ Other & $0.64(0.35-1.17)$ & 0.15 \\
Partner status (Reference $=$ Partner/Spouse) & $1.45(0.89-2.35)$ & 0.14 \\
Lack of participation in regular exercise & $1.66(1.05-2.65)$ & 0.032 \\
Current smoking & $1.35(0.72-2.51)$ & 0.35 \\
Estimated glomerular filtration rate $<60 \mathrm{ml} / \mathrm{min} / 1.73^{2}$ & $1.20(0.66-2.18)$ & 0.55 \\
Poor sleep quality (PSQI $\geq 5)$ & $1.30(0.75-2.25)$ & 0.35 \\
Charlson comorbidity index $a$ & $1.32(1.02-1.70)$ & 0.03 \\
Grace risk score $a$ & $1.34(0.76-2.36)$ & 0.25 \\
Bodily pain $a, b$ & $0.61(0.47-0.77)$ & $<0.0001$ \\
Current depressive disorder & $1.94(1.01-3.73)$ & 0.048 \\
Probable anxiety disorder $(\mathrm{HADS}-\mathrm{A} \geq 8)$ & $2.00(0.81-4.97)$ & 0.13 \\
\hline
\end{tabular}

${ }^{a}$ Per standard deviation increase;

$b_{\text {Higher scores indicate less bodily pain. }}$

Abbreviations. PSQI = Pittsburgh Sleep Quality Inventory; HADS-A, Hospital Anxiety and Depression Scale-Anxiety subscale. 